\title{
Effects of Composition on Structural and Magnetic Properties of Free-Standing Single Crystal Ferromagnetic Shape Memory Ni-Mn-Ga Films Grown by Molecular Beam Epitaxy
}

\author{
S. McKernan", T.C. Shih", J.W. Dong", J.Q. Xie ${ }^{*}$, X.Y. Dong", C.J. Palmstrøm", and R. D. James ${ }^{* *}$ \\ * Department of Chemical Engineering and Material Science, University of Minnesota, Minneapolis, \\ MM 55455; \\ ** Department of Aerospace Engineering and Mechanics, University of Minnesota, Minneapolis, \\ MN 55455
}

Ferromagnetic shape memory alloys possess the potential for producing mechanical actuators when used in combination with semiconductor devices. Single crystal films of Ni-Mn-Ga ferromagnetic shape memory alloys in particular may become extremely productive since they can be grown epitaxially on GaAs-based semiconductor devices. The ferromagnetic properties of Ni-Mn-Ga alloys are extremely sensitive to the alloy composition [1]. Ni-Mn-Ga alloys have been grown as epilayers using molecular beam epitaxy to produce various alloy compositions with varying valence electron concentration, e/a [2]. Structures consisting of GaAs/AlGaAs/GaAs heterostructures on GaAs (001) substrates were grown prior to the Ni-Mn-Ga alloy growth. This procedure was used to give a clean and smooth starting surface for the epitaxial Ni-Mn-Ga growth, and to aid in the etching procedure used to prepare free-standing shape memory alloy films. The Ni-Mn-Ga epilayer films were patterned as cantilevers and bridges along specific crystallographic directions and released from the GaAs substrate by wet chemical etching as is shown in Figure 1.

The martensitic transformation that occurs upon cooling was followed in a variety of instruments. The magnetic properties of the films were determined by means of vibrating sample magnetometer and superconductor quantum interference devices measurements before and after release from GaAs substrates. Rutherford backscattering spectrometry, X-ray diffraction, and transmission electron microscopy were used for composition and structural measurements. The highest Curie temperature $(\sim 390 \mathrm{~K})$ and saturation magnetization at room temperature $\left(\sim 600 \mathrm{emu} / \mathrm{cm}^{3}\right)$ were observed for the films with composition close to $\mathrm{Ni}_{50} \mathrm{Mn}_{30} \mathrm{Ga}_{20}$. This corresponds to an e/a of $\sim 7.70$. Increasing or decreasing the e/a ratio resulted in both Curie temperature and saturation magnetization decreasing. Martensitic phase transformation was only observed for released films.

In this presentation, the effects of composition will be correlated with the magnetic properties and martensitic phase transformation in single crystal epitaxial Ni-Mn-Ga films before and after substrate removal. For intact films that span the window in the substrate this results in a "tenting" of the film as it goes through the martensitic phase transformation, and for films that have only one connected edge, more dynamic effects are seen. The transition can be followed in the optical microscope and in the SEM as a function of temperature. By patterning the films parallel to and at $45^{\circ}$ to the $\{100\}$ GaAs substrate planes, the influence of crystallography is assessed, and influences of the confinement of the film are assessed by varying the aspect ratio of the released film. [3]

\section{References}

1. V. A. Chernenko; Scropta Materialia; 40, 523 (1999)

2. J. W. Dong, et. al.; J. Appl. Phys. 88, 7357 - 7359 (2000) 
3. This research was supported in part by the MRSEC program of the NSF under Award No. DMR9809362 and by ONR Contract No. N/N00014-01-1-0830.

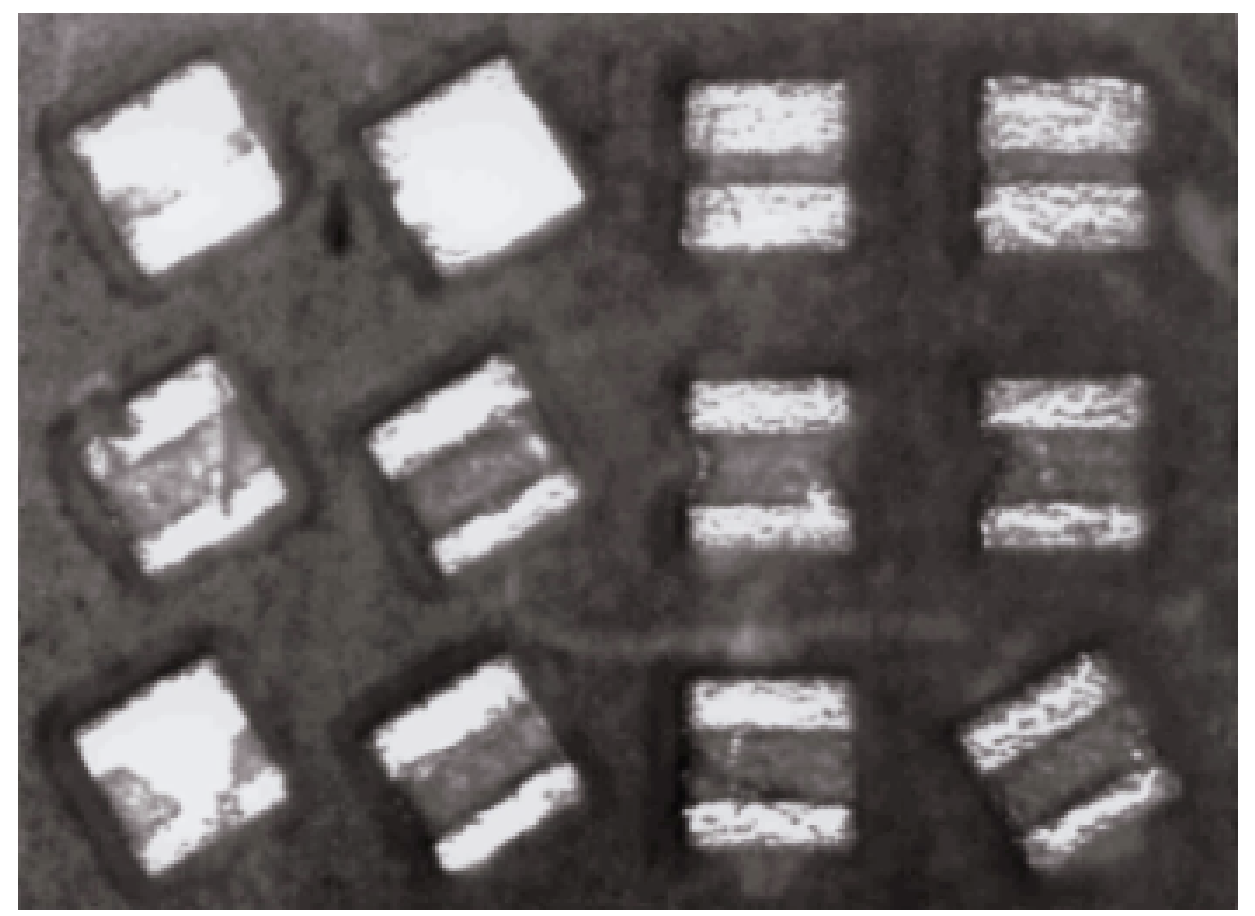

Figure 1 Optical micrographs of different aspect ratio free-standing shape memory alloy films. Note that different elements are patterned at $45^{\circ}$ to each other to provide data on different crystallographic orientations.

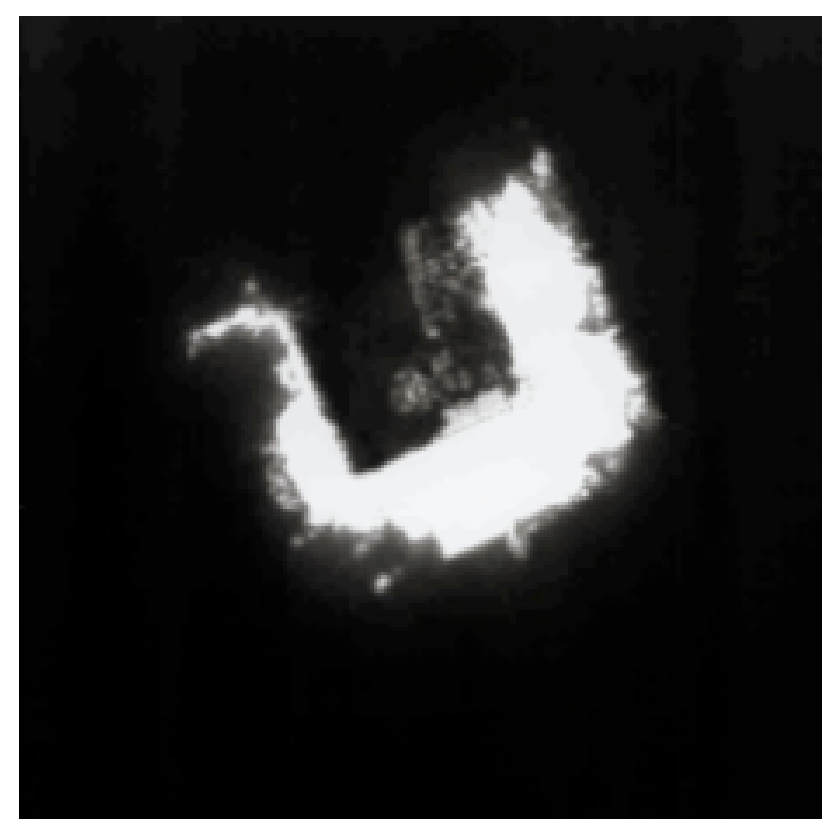

Figure 2 Higher magnification image of the released films. 